\title{
An Exploration of the Impact of Anticentromere Antibody on Early-Stage Embryo
}

\author{
Ying Ying, ${ }^{1}$ Xi Guo, ${ }^{2}$ Yiping Zhong, ${ }^{2}$ and Canquan Zhou ${ }^{2}$ \\ ${ }^{1}$ Reproductive Medicine Center, The Third Affiliated Hospital of Guangzhou Medical University, Guangzhou, China \\ ${ }^{2}$ Reproductive Medicine Center, The First Affiliated Hospital of Sun Yat-sen University, Guangzhou, China
}

Correspondence should be addressed to Yiping Zhong; zypdoctor@126.com and Canquan Zhou; zhoucanquan@hotmail.com

Received 11 June 2017; Accepted 16 July 2017; Published 4 October 2017

Academic Editor: Jacek Tabarkiewicz

Copyright (c) 2017 Ying Ying et al. This is an open access article distributed under the Creative Commons Attribution License, which permits unrestricted use, distribution, and reproduction in any medium, provided the original work is properly cited.

\begin{abstract}
Background. Previously, we found women with positive anticentromere antibody showed impaired potential of oocyte maturation and embryo cleavage; the possible mechanism behind this phenomenon was still unknown. Objective. Thus, the present study aimed to preliminarily explore whether ACA could penetrate into the living embryos and impair their developmental potential via in vitro coculture with mouse embryos. Methods. Mouse embryos were collected and used for in vitro culture with polyclonal anticentromere protein A (CENP-A) antibody; then, immunofluorescence assay was performed to determine the penetration of antibody into embryos, and embryo development potential was observed. Results. All embryos cultured with anti-CENP-A antibody exhibited immunofluorescence on the nucleus, while none of the embryos from the control groups showed immunofluorescence. Additionally, embryos cultured with anti-CENP-A antibody experienced significant growth impairment compared with controls. Conclusion. Mouse embryos may be a direct target for ACA in vitro prior to implantation. However, the precise mechanism needs further clarification.
\end{abstract}

\section{Introduction}

A recent study revealed disorders of oocyte maturation and early embryonic development in women with positive anticentromere antibody (ACA) in their peripheral blood [1] More recently, we found that women positive for ACA had a significantly lower percentage of mature oocytes and embryo cleavage rate compared with women negative for ACA [2], further revealing the potential impact of ACA on female fertility. ACA is known to be one of the members of ANAs. It was first discovered in 1980 as a specific antibody against centromere in serum of patients with calcinosis, Raynaud's phenomenon, esophageal dysmotility, sclerodactyly, and telangiectasia (CREST) syndrome $[3,4]$. Now, ACA has been recognized as an effective auxiliary diagnostic marker for systemic sclerosis (SSc). As reported, female patients with SSc are susceptible to have several different adverse pregnancy outcomes, including increased spontaneous abortion rate, premature birth, small babies, and infertility $[5,6]$. Additionally, the infertility prevalence in patients with SSc is high, and the success rate for infertility treatment is relatively low, which needs further investigation [7].

As early as the 1990s, researchers attempted to microinject ACA into mouse eggs, which led to disorders of chromosomal movement and segregation [8]. It is known that kinetochore is the attachment site of spindle microtubules in the centromeric region of a chromosome $[9,10]$. Also, it is the dynamic structure for mitosis, meiosis, and other important activities of cells [11-15]. Therefore, it would be reasonable to infer that ACA might interfere with meiosis or mitosis in living cells.

Centromere is a DNA-protein complex, and its assembly is coregulated by centromeric chromatins and their associated protein complex $[16,17]$. Centromere protein A (CENP-A) is one of the constitutive centromere proteins with relatively clear biological functions that has been mostly studied; its important role in assembly and functional implementation of centromere has been repeatedly verified $[18,19]$. Furthermore, similar to CENP-B, CENP-A is considered to be a major target antigen of ACA [20-23]. 
It was speculated that ACA might be one of the ANAs most closely associated with abnormal oocyte maturation and embryo cleavage. Therefore, the aim of the present study was to explore the potential impact of ACA on early-stage embryos via in vitro coculture with mouse embryos.

\section{Materials and Methods}

2.1. Mouse Embryos. Superovulation was induced in outbred ICR mice by stimulating with pregnant mare's serum gonadotrophin (10 IU intraperitoneally (i.p.)) and human chorionic gonadotrophin (10 IU i.p. after $48 \mathrm{~h}$ ) and mated with ICR males. The female mice were killed $24 \mathrm{~h}$ after mating. Early-stage embryos were collected by sharp dissection of the fallopian tubes and used in the experiments. The Ethics Committee of the First Affiliated Hospital of Sun Yat-Sen University approved this study.

2.2. In Vitro Embryo Culture. The embryos were cultured in the Quinn's serial medium (Sage, USA). For the antibody group, rabbit polyclonal antibody to mouse CENP-A (bovine serum albumin and azide free, customized products from Abcam, United Kingdom) was added to the medium. The antibody concentration in the medium was $35 \mu \mathrm{g} / \mathrm{mL}$ (modified based on the literature [24]). For the phosphate-buffered saline (PBS) group (served as controls), the PBS solution (PBS tablet, Millipore, Merck, Germany) with the same volume as the antibody solution was added to the medium. The blank control group comprised the medium without any additives.

2.3. Immunofluorescence Assay. On the second and third days of culture, three to five embryos were picked from each dish of the three groups for the immunofluorescence assay, to detect whether the signals of anti-CENP-A antibody were present in the embryos after coculture. The procedures for the immunofluorescence assay were as follows: The embryos were fixed in $4 \%$ polyoxymethylene and then permeated with $0.5 \%$ Triton X-100 (Sigma, USA), followed by sealing in 5\% normal donkey serum (Jackson Immunoresearch, USA). After that, the embryos were incubated for $1 \mathrm{~h}$ with 488-labeled donkey antirabbit IgG (Invitrogen, UK, $1: 1000$ dilution), rinsed, incubated with $1 \mu \mathrm{g} / \mathrm{mL}$ DAPI (4',6-diamidino-2-phenylindole, dihydrochloride, Cell Signaling Technology, USA) for $15 \mathrm{~min}$, again rinsed, and fixed in a dish for subsequent microscopic observation. In order to rule out false positive of the experimental group, embryos from antibody group were incubated with PBS instead of 488labeled donkey antirabbit IgG (antibody group for control).

2.4. Development of Cocultured Embryos (Embryotoxicity Assay). The collection and culturing of the embryos for this embryotoxicity assay were the same as described earlier except that the embryos remained in the dishes for the entire 3-day period. The embryos in each group were examined to determine their stage of development on the third and fifth days (the first day referred to the day of oocyte collection). The following developmental stages were recorded: 6- to 8-cell stages on the third day, and blastocyst, morula, 2- to 8-cell, and atretic stages on the fifth day.
2.5. Statistical Analysis. Statistical analysis was performed using SPSS 13 (SPSS, IL, USA). A chi-square test and partition of chi-square tests were used to compare qualitative data. A $P$ value less than 0.05 was considered statistically significant by chi-square test among the three groups, and a $P$ value less than 0.0167 was used to indicate statistical significance in the partition of chi-square tests between groups.

\section{Results}

3.1. Immunofluorescence. All embryos cultured with antiCENP-A antibody exhibited strong immunofluorescence in their nuclei, while none of the embryos from the PBS and blank control groups, as well as the antibody group for control, showed immunofluorescence (Figure 1).

3.2. Embryotoxicity Assay. Compared with the PBS and blank control groups, the percentages of 6- to 8-cell stage embryos on the third day, as well as blastula and morula stage embryos on the fifth day, were significantly lower in the antibody group. The developmental potentials of embryos were comparable between the PBS and blank control groups (Table 1).

\section{Discussion}

In 1999, researchers found that all mouse embryos cultured with purified antinuclear IgG exhibited strong immunofluorescence on the embryonic cells and experienced significant growth impairment or death compared with those cultured with control immunoglobulins [25]. This indicated that ANAs could bind directly to embryos in vitro. However, the precise epitopes were not known because no nuclear antigens or phospholipids were found in the zona. In mouse 2-cell stage embryos, a set of nucleoproteins is transiently synthesized and changes in embryonic chromatin composition, suggesting that early embryos may possess epitopes for ANA [26]. Furthermore, the binding is relatively specific, as antithyroid antibody and antibodies from healthy individuals show no evidence of binding to embryos. Microinjection of serum containing ACA into mouse oocytes could hinder chromosome congression and cause meiotic arrest in interphase or mitotic arrest in prometaphase [8].

In the present study, all embryos cultured with polyclonal anti-CENP-A antibody showed strong immunofluorescence of antibody against nuclear components (which were speculated to be anti-CENP-A antibody) and experienced apparent embryonic growth impairment, indicating that mouse embryos may be a direct target for some ACAs in vitro prior to implantation. In addition, for the majority of cocultured embryos, always only one or some of the blastomeres showed fluorescence. Perhaps, the density of structures in and around the centromere prevents anti-CENP-A antibody accessibility, or the blastomere with detectable fluorescence was inclined to apoptosis and displayed relatively loose structures that enabled anti-CENP-A antibody accessibility. However, the precise mechanism needs further clarification.

Although no definite concept exists for antibody entering the living cells, and the mechanism involved is unknown yet, these studies provided evidence for antibodies entering the 


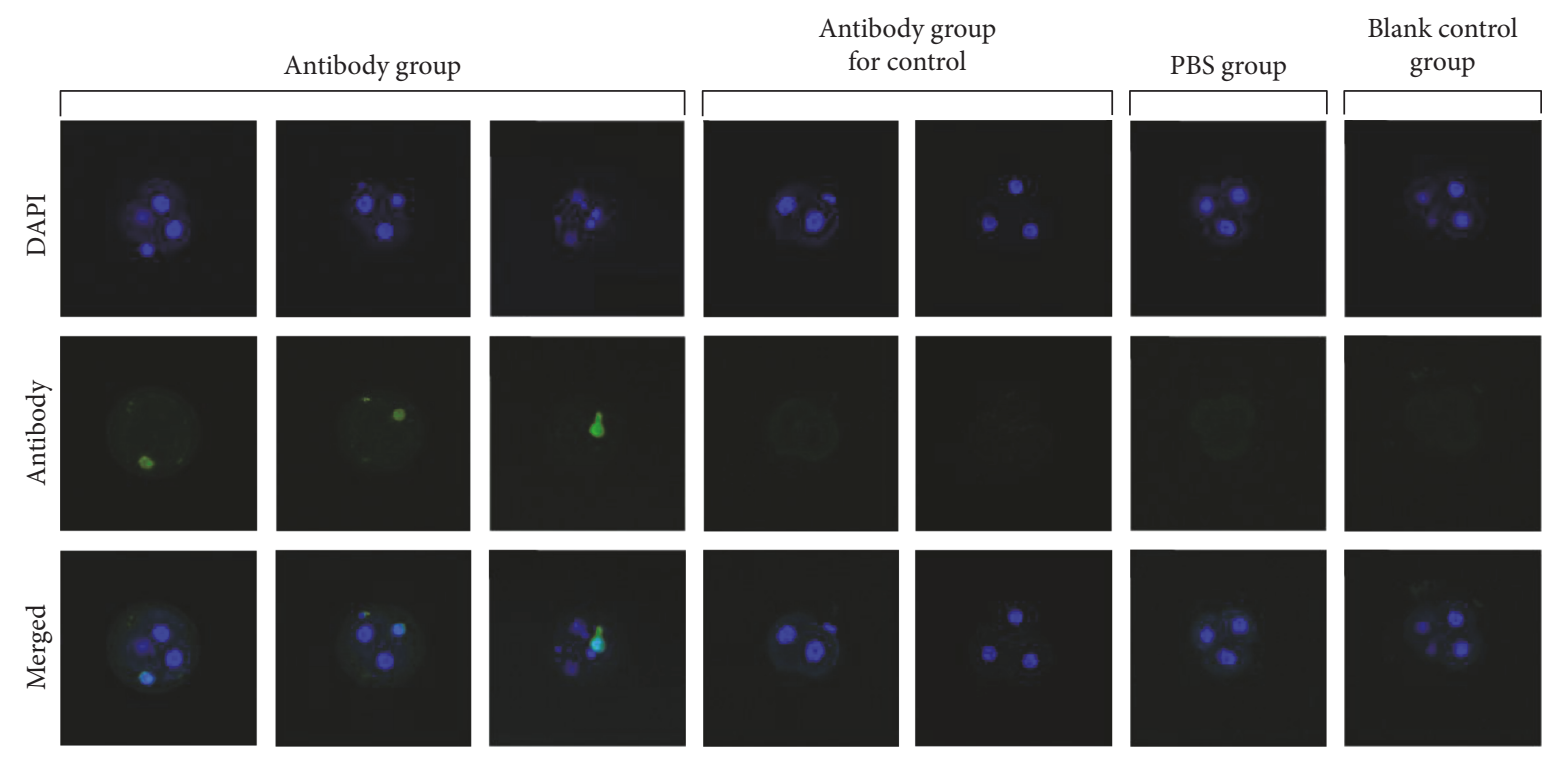

FIGURE 1: Immunofluorescence views of embryos cultured with anti-CENP-A antibody (original magnification $\times 400$ ). Antibody fluorescence was present on the nuclei of blastomeres in embryos from the antibody group, while no significant antibody fluorescence was observed in the embryos from groups of PBS, blank control, and antibody group for control.

TABLe 1: Embryotoxicity assay.

\begin{tabular}{|c|c|c|c|c|}
\hline Parameters (\%) & Antibody group & PBS group & Blank control group & $P$ value \\
\hline 6- to 8-cell stages on the third day & $24.8 \%(114 / 459)$ & $48.4 \%(183 / 378)$ & $51.0 \%(192 / 376)$ & $<0.01^{\mathrm{a}, \mathrm{b}}$ \\
\hline Blastula stage on the fifth day & $26.8 \%(123 / 459)$ & $57.1 \%(216 / 378)$ & $64.9 \%(244 / 376)$ & $<0.01^{\mathrm{a}, \mathrm{b}}$ \\
\hline Morula stage on the fifth day & $12.4 \%(57 / 459)$ & $24.6 \%(93 / 378)$ & $20.2 \%(76 / 376)$ & $<0.01^{\mathrm{a}, \mathrm{b}}$ \\
\hline
\end{tabular}

The first day referred to the day of embryo collection. $P<0.05$ was considered statistically significant among the three groups. ${ }^{a} P<0.0167$ versus antibody and PBS groups. ${ }^{b} P<0.0167$ versus antibody and blank control groups.

living cells. For example, the anti-ribonucleoprotein-IgG could selectively enter the T-lymphocytes, while relatively less Fc receptors were present on the surface of $\mathrm{T}$ lymphocytes. It suggested that some other mechanisms relevant to non-Fc regulation might be involved, which might be associated with antigen-like structures on the lymphocyte surface, implying that the ribonucleoprotein antibodies interacted with the ribonucleoprotein antigens on the cell surface to regulate their access into the living cells [27].

This study also found that the embryonic developmental potential was significantly impaired after culture with anti-CENP-A antibody, exhibiting significantly lower percentages of 6- to 8-cell stage embryos on the third day and blastula stage embryos on the fifth day. In fact, a previous study found that mouse embryos cultured with purified IgG from ANA-positive serum showed significantly impaired embryonic development potential [25]. ANA could penetrate into subcellular structures containing corresponding antigens, where it could identify and bind to epitopes in the important functional regions. These autoantibodies could inhibit significantly the function of antigens both in vivo and in vitro [28].

In conclusion, embryos cultured with anti-CENP-A antibody experienced significant growth impairment or death. Thus, embryos could be a direct target for antiCENP-A antibody.

\section{Ethical Approval}

Medical Ethics Committee of The First Affiliated Hospital of Sun Yat-sen University approved the study (no. [2014]75).

\section{Conflicts of Interest}

Ying Ying, Xi Guo, Yiping Zhong, and Canquan Zhou declare that they have no conflict of interest.

\section{Acknowledgments}

This study was supported by the National Natural Science Foundation of China (no. 81701518), Guangzhou Science and Technology and Information Bureau (no. 2014J4100161), Science \& Technology Project of Guangdong Province (no. 2013B021800271), and Population and Family Planning Commission of Guangdong Province (no. 20132048). This study was supported by the Key Laboratory for Reproductive Medicine of Guangdong Province from The First Affiliated Hospital of Sun Yat-sen University. The study 
was also supported by two key laboratories from The Third Affiliated Hospital of Guangzhou Medical University; they were the Key Laboratory for Major Obstetric Diseases of Guangdong Province and Key Laboratory of Reproduction and Genetics of Guangdong Higher Education Institutes.

\section{References}

[1] Y. Nagata, K. Honjou, H. Tsujioka, T. Yoshizato, and S. Miyamoto, "Involvement of anticentromere antibody in interference with oocyte meiosis and embryo cleavage," Fertility and Sterility, vol. 95, pp. 2729-2731, 2011.

[2] Y. Ying, Y. P. Zhong, C. Q. Zhou et al., "Preliminary investigation of the impact of anticentromere antibody on oocyte maturation and embryo cleavage," Fertility and Sterility, vol. 100, pp. 1585-1589, 2013.

[3] Y. Moroi, C. Peebles, M. J. Fritzler, J. Steigerwald, and E. M. Tan, "Autoantibody to centromere (kinetochore) in scleroderma sera," Proceedings of the National Academy of Sciences of the United States of America, vol. 77, pp. 1627-1631, 1980.

[4] E. M. Tan, G. P. Rodnan, I. Garcia, Y. Moroi, M. J. Fritzler, and C. Peebles, "Diversity of antinuclear antibodies in progressive systemic sclerosis. Anti-centromere antibody and its relationship to CREST syndrome," Arthritis and Rheumatism, vol. 23, pp. 617-625, 1980.

[5] A. J. Silman and C. Black, "Increased incidence of spontaneous abortion and infertility in women with scleroderma before disease onset: a controlled study," Annals of the Rheumatic Diseases, vol. 47, pp. 441-444, 1988.

[6] C. M. Black and W. M. Stevens, "Scleroderma," Rheumatic Diseases Clinics of North America, vol. 15, pp. 193-212, 1989.

[7] V. D. Steen and T. A. Medsger Jr., "Fertility and pregnancy outcome in women with systemic sclerosis," Arthritis and Rheumatism, vol. 42, pp. 763-768, 1999.

[8] C. Simerly, R. Balczon, B. R. Brinkley, and G. Schatten, "Microinjected centromere [corrected] kinetochore antibodies interfere with chromosome movement in meiotic and mitotic mouse oocytes," The Journal of Cell Biology, vol. 111, pp. 1491-1504, 1990.

[9] C. L. Rieder, "The formation, structure, and composition of the mammalian kinetochore and kinetochore fiber," International Review of Cytology, vol. 79, pp. 1-58, 1982.

[10] B. R. Brinkley, M. M. Valdivia, A. Tousson, and R. D. Balczon, "The kinetochore: structure and molecular characterization," in Mitosis: Molecules and Mechanisms, J. Hyams and B. R. Brinkley, Eds., Academic Press, New York, NY, USA, 1989.

[11] R. B. Nicklas, "Chromosomes and kinetochores do more in mitosis than previously thought," in Chromosome Structure and Function, Stadler Genetics Symposia Series, J. P. Gustafson and R. Appels, Eds., Springer, Boston, MA, USA, 1988.

[12] T. J. Mitchison, "Microtubule dynamics and kinetochore function in mitosis," Annual Review of Cell Biology, vol. 4, pp. 527-549, 1988.

[13] T. J. Mitchison, "Mitosis: basic concepts," Current Opinion in Cell Biology, vol. 1, pp. 67-74, 1989.

[14] T. J. Mitchison and M. W. Kirschner, "Properties of the kinetochore in vitro. I. Microtubule nucleation and tubulin binding," The Journal of Cell Biology, vol. 101, pp. 755-765, 1985.
[15] R. B. Nicklas, "The motor for poleward chromosome movement in anaphase is in or near the kinetochore," The Journal of Cell Biology, vol. 109, pp. 2245-2255, 1989.

[16] M. Glynn, A. Kaczmarczyk, L. Prendergast, N. Quinn, and K. F. Sullivan, "Centromeres: assembling and propagating epigenetic function," Sub-Cellular Biochemistry, vol. 50, pp. 223-249, 2010.

[17] Y. Cai, Y. Weng, and R. Yang, "Progress on chromosome centromere-specific proteins," Foreign Medical Genetics Volume, vol. 28, pp. 196-199, 2005.

[18] L. Huang and J. Xia, "Advances in the study of structure and function of human centromere," Foreign Medical Genetics Volume, vol. 23, pp. 1-4, 2000.

[19] L. Kremer, J. M. Alvaro-Gracia, C. Ossorio, and J. Avila, "Proteins responsible for anticentromere activity found in the sera of patients with CREST-associated Raynaud's phenomenon," Clinical and Experimental Immunology, vol. 72, pp. 465-469, 1988.

[20] S. L. Pfueller, D. Logan, T. T. Tran, and R. A. Bilston, "Naturally occurring human IgG antibodies to intracellular and cytoskeletal components of human platelets," Clinical and Experimental Immunology, vol. 79, pp. 367-373, 1990.

[21] M. J. Fritzler, J. D. Pauls, T. D. Kinsella, and T. J. Bowen, "Antinuclear, anticytoplasmic, and anti-Sjogren's syndrome antigen A (SS-A/Ro) antibodies in female blood donors," Clinical Immunology and Immunopathology, vol. 36, pp. 120-128, 1985.

[22] K. Aho, P. Koskela, R. Makitalo, M. Heliovaara, and T. Palosuo, "Antinuclear antibodies heralding the onset of systemic lupus erythematosus," The Journal of Rheumatology, vol. 19, pp. 1377-1379, 1992.

[23] O. J. Stone, "Autoimmunity as a secondary phenomenon in scleroderma (and so-called human adjuvant disease)," Medical Hypotheses, vol. 34, pp. 127-130, 1991.

[24] K. Yanase and M. P. Madaio, "Nuclear localizing anti-DNA antibodies enter cells via caveoli and modulate expression of caveolin and p53," Journal of Autoimmunity, vol. 24, pp. 145-151, 2005.

[25] B. D. Kaider, C. B. Coulam, and R. G. Roussev, "Murine embryos as a direct target for some human autoantibodies in vitro," Human Reproduction, vol. 14, pp. 2556-2561, 1999.

[26] H. J. Clarke, "Nuclear and chromatin composition of mammalian gametes and early embryos," Biochemistry and Cell Biology, vol. 70, pp. 856-866, 1992.

[27] J. Ma, N. King, S. L. Chen, R. Penny, and S. N. Breit, “Antibody penetration of viable human cells. II. Anti-RNP antibodies binding to RNP antigen expressed on cell surface, which may mediate the antibody internalization," Clinical and Experimental Immunology, vol. 93, pp. 396-404, 1993.

[28] E. M. Tan, Y. Muro, and K. M. Pollard, "Autoantibody-defined epitopes on nuclear antigens are conserved, conformationdependent and active site regions," Clinical and Experimental Rheumatology, vol. 12, Supplement 11, pp. S27-S31, 1994. 


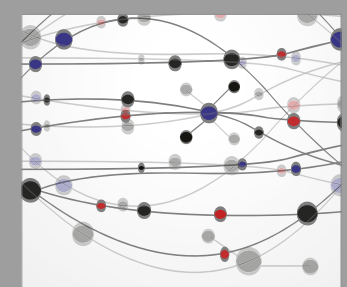

The Scientific World Journal
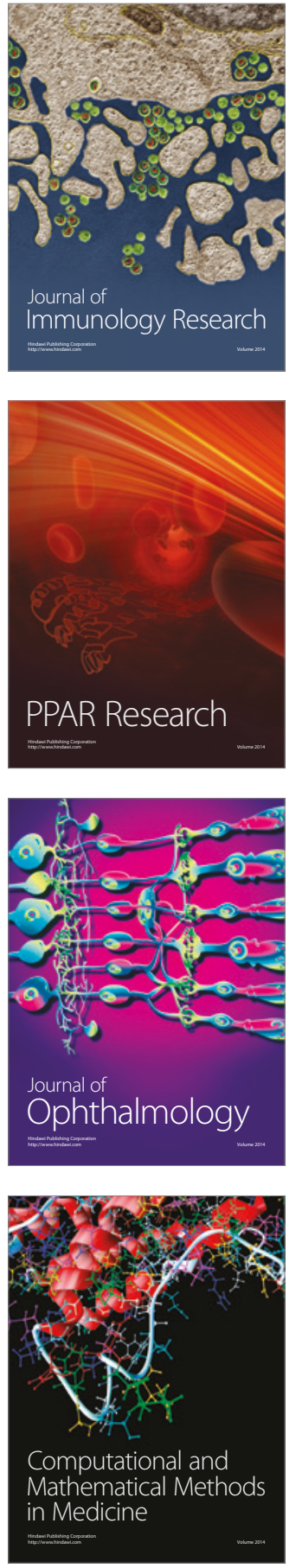

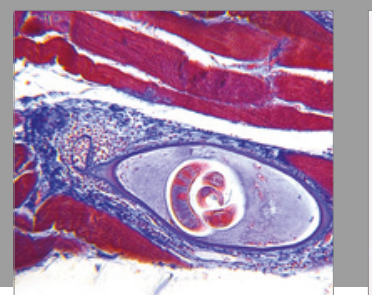

Gastroenterology Research and Practice
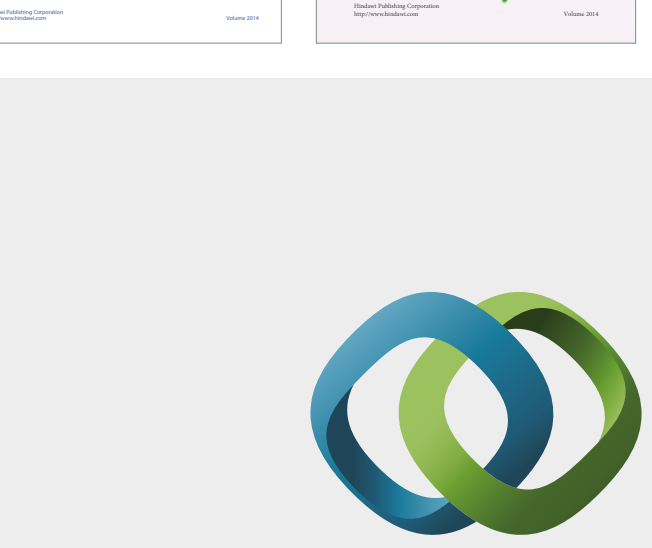

\section{Hindawi}

Submit your manuscripts at

https://www.hindawi.com
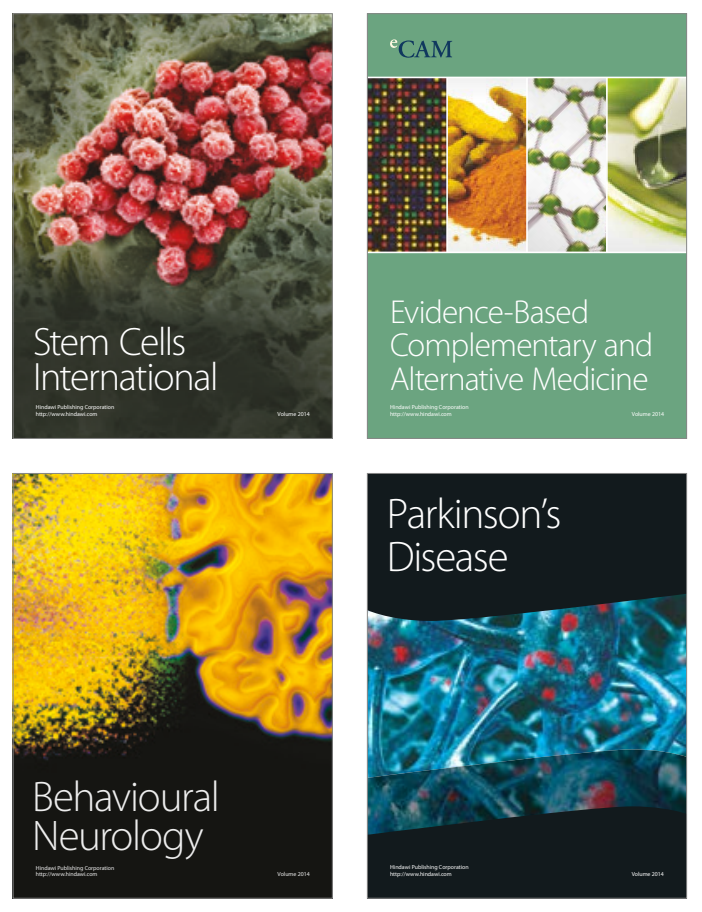
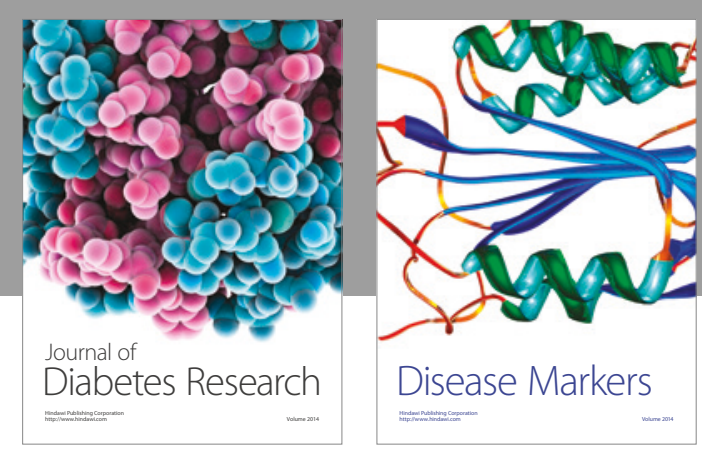

Disease Markers
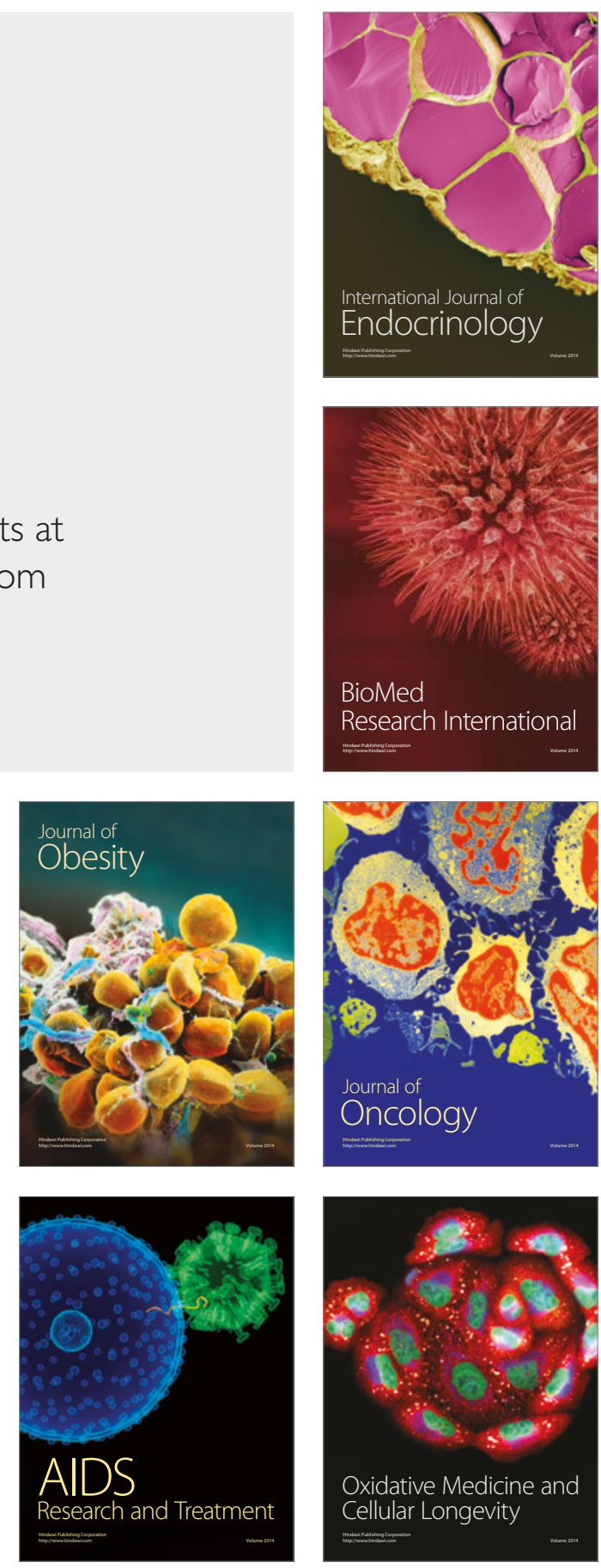